\title{
Energetics and Dynamics of Proton-Coupled Electron Transfer in the NADH/FMN Site of Respiratory Complex I
}

\author{
Patricia Saura $^{\dagger}$ and Ville R. I. Kaila* ${ }^{* \dagger}$ \\ ${ }^{\dagger}$ Department of Chemistry, Technical University of Munich (TUM), Lichtenbergstrasse 4, Garching D-85747, Germany
}

Supporting Information

\begin{abstract}
Complex I functions as an initial electron acceptor in aerobic respiratory chains that reduces quinone and pumps protons across a biological membrane. This remarkable charge transfer process extends ca. $300 \AA$ and it is initiated by a poorly understood proton-coupled electron transfer (PCET) reaction between nicotinamide adenine dinucleotide $(\mathrm{NADH})$ and a protein-bound flavin (FMN) cofactor. We combine here large-scale density functional theory calculations and quantum/classical models with atomistic molecular dynamics simulations to probe the energetics and dynamics of the NADH-driven PCET reaction in complex I. We find that the reaction takes place by concerted hydrogen atom $\left(\mathrm{H}^{\bullet}\right)$ transfer that couples to an electron transfer $(\mathrm{eT})$ between the aromatic ring systems of the cofactors and further triggers reduction of the nearby FeS

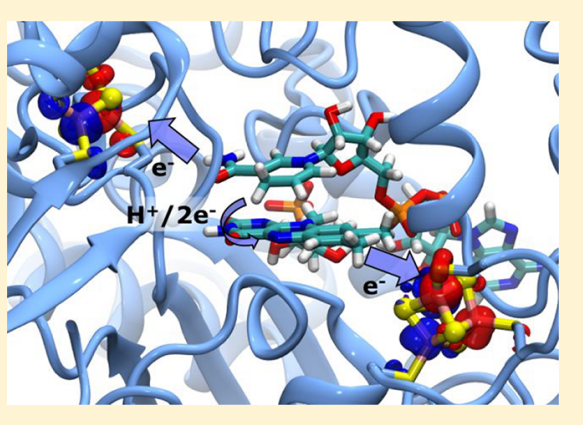
centers. In bacterial, Escherichia coli-like complex I isoforms, reduction of the Nla FeS center increases the binding affinity of the oxidized $\mathrm{NAD}^{+}$that prevents the nucleotide from leaving prematurely. This electrostatic trapping could provide a protective gating mechanism against reactive oxygen species formation. We also find that proton transfer from the transient FMNH ${ }^{\bullet}$ to a nearby conserved glutamate (Glu97) residue favors eT from Nla onward along the FeS chain and modulates the binding of a new NADH molecule. The PCET in complex I isoforms with low-potential Nla centers is also discussed. On the basis of our combined results, we propose a putative mechanistic model for the NADH-driven proton/electron-transfer reaction in complex I.
\end{abstract}

\section{INTRODUCTION}

Complex I (NADH:ubiquinone oxidoreductase) functions as the electron entry point in aerobic respiratory chains. It catalyzes electron transfer $(\mathrm{eT})$ between nicotinamide adenine dinucleotide $(\mathrm{NADH})$ and quinone $(\mathrm{Q})$, which the enzyme employs to pump protons across a biological membrane. ${ }^{1-5}$ This generates a proton motive force $(p m f)$ that thermodynamically drives synthesis of adenosine triphosphate (ATP) by $\mathrm{F}_{1} \mathrm{~F}_{\mathrm{o}}$-ATPase and active transport. ${ }^{6,7}$ The eT process is fully reversible, and under high pmf conditions, complex I catalyzes the reverse eT from quinol $\left(\mathrm{QH}_{2}\right)$ to $\mathrm{NAD}^{+} ., 9$ Such operation modes are physiologically relevant under hypoxic conditions, where electrons leak out from complex I. ${ }^{10}$ The eT in complex I takes place in the hydrophilic domain, which extends ca. 100 $\AA$ above the membrane plane, whereas the proton pumping machinery comprises the ca. $200 \AA$ wide membrane domain. It currently remains unclear how these two processes are coupled across such large distances, but mechanistic models have recently started to emerge (see ref 5 and refs therein).

The NADH binding site is located in a solvent-accessible cavity of the Nqo1 subunit (Thermus thermophilus nomenclature) at the "upper edge" of the hydrophilic domain (Figure 1). ${ }^{11,12}$ This cavity contains a flavin mononucleotide (FMN) cofactor that is surrounded by an unusual Rossmann fold with four parallel $\beta$-strands in contrast to the six strands typically found in such motifs. This modification could have evolved to allow for binding of the two nucleotides and to ensure a tight packing of the aromatic ring systems to facilitate the charge transfer between NADH and FMN that is supported by several conserved residues in the cavity. ${ }^{13}$

The first step in complex I catalysis is the oxidation of $\mathrm{NADH}$ at a high rate of ca. $600-15000 \mathrm{~s}^{-1,14,15}$ which involves transfer of two electrons via a proton-coupled electron transfer (PCET) process to the FMN, most likely forming $\mathrm{FMNH}^{-}$or $\mathrm{FMNH}_{2}$, whereas no stable flavosemiquinone radical $\left(\mathrm{FMNH}^{\circ}\right)$ species has so far been detected. ${ }^{16}$ The process effectively involves a hydride ion $\left(\mathrm{H}^{-}=\mathrm{H}^{+} / 2 \mathrm{e}^{-}\right)$ transfer, although the exact reaction intermediates remain unknown. The reaction is thus chemically similar to many organic and enzyme-catalyzed PCET reactions. ${ }^{17-20}$

After the flavin reduction, the two electrons enter the $\mathrm{FeS}$ chain to stepwise reduce $\mathrm{Q}$ to $\mathrm{QH}_{2}$ that links to local proton transfer from His38 and Tyr87 of the Nqo4 subunit. ${ }^{21,22}$ The $\mathrm{FeS}$ centers are one-electron carriers in complex $\mathrm{I}^{23}$ and the $\mathrm{NADH} / \mathrm{FMN}$ site has been suggested based on experiments in Escherichia coli to function as a two-to-one electron converter that bifurcates the eT process in the FeS chain. ${ }^{15}$ To this end, $\mathrm{N} 1 \mathrm{a}$ and N3, which are located at 6-10 $\AA$ from the NADH/ FMN site, function as the initial electron acceptors (Figure 1A, left inset). The $\mathrm{N} 3$ center mediates the eT across the ca. $100 \AA$ $\mathrm{FeS}$ wire that connects FMN with $\mathrm{Q}$ (Figure 1A, right inset).

Received: October 14, 2018

Published: March 15, 2019 

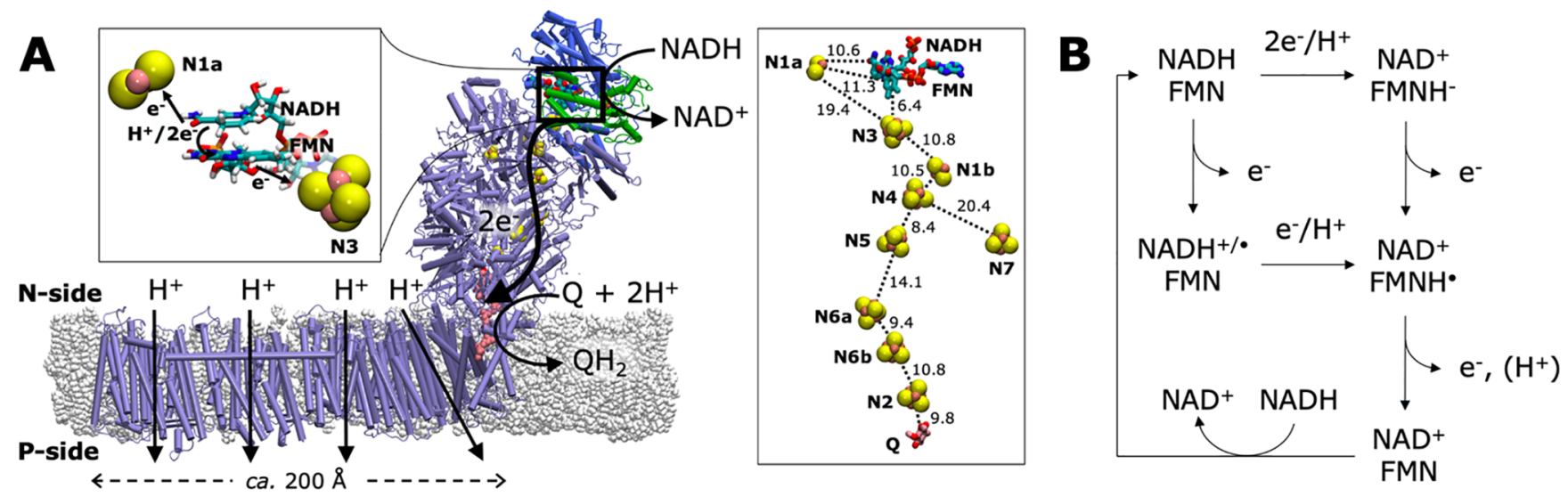

Figure 1. (A) Structure of the bacterial complex I (PDB ID: 4HEA) from T. thermophilus. Electrons are transferred from NADH to Q in the hydrophilic domain, and the released free energy is employed in pumping protons across the membrane domain. Left inset: The NADH/FMN binding site from the NADH-bound crystal structure (PDB ID: 3IAM), showing NADH, FMN, the N3 FeS center of Nqo1 (in blue), and the N1a $\mathrm{FeS}$ center of Nqo2 (in green). Right inset: FeS centers connecting the NADH/FMN site with Q (modeled in its cavity), showing edge-to-edge distances between the centers. (B) Putative reaction intermediates in the PCET reaction between NADH and FMN in complex I. The vertical bars indicate oxidation by nearby $\mathrm{FeS}$ centers, and horizontal bars indicate coupled $\mathrm{H}^{+} / \mathrm{e}^{-}$-transfer reactions.

The exact role of N1a, which is located on the side of the main transfer pathway, remains unclear. The N1a redox potential is unknown for the mammalian complex I, but a redox potential as low as $-400 \mathrm{mV}$ has been indirectly suggested for some species, ${ }^{24,25}$ relative to the redox potential of the other $\mathrm{FeS}$ centers at ca. $-320 \mathrm{mV}$ and the terminal N2 center at ca. -200 $\mathrm{mV}^{26,27}$ In contrast, reduction of N1a in E. coli complex I, with an $E_{\mathrm{m}}$ of $-240 \mathrm{mV}$, has been observed in EPR experiments. ${ }^{15,28}$

Nla could have a protective role against formation of reactive oxygen species (ROS) that is linked to development of mitochondrial disorders, ${ }^{9,10,29-31}$ by modulating the dissociation of $\mathrm{NAD}^{+}$from the FMN site. ${ }^{31}$ Interestingly, the relative $K_{\mathrm{M}}$ of $\mathrm{NADH}$ and $\mathrm{NAD}^{+}$depends on the direction of the eT process: the $K_{\mathrm{M}}(\mathrm{NADH})$ is 2 orders of magnitude lower than the $K_{\mathrm{i}}\left(\mathrm{NAD}^{+}\right)$in the $1 \mathrm{mM}$ range during forward eT, whereas under reverse eT conditions from $\mathrm{QH}_{2}$ to $\mathrm{NAD}^{+}, K_{\mathrm{M}}\left(\mathrm{NAD}^{+}\right)$ is in the $10-20 \mu \mathrm{M}$ range. ${ }^{3,13,32-34}$ Moreover, the oxidized $\mathrm{NAD}^{+}$is released on the millisecond time scale. ${ }^{15}$

To probe the energetics and mechanism of the initial PCET process between $\mathrm{NADH}$ and $\mathrm{FMN}$, and its coupling to subsequent eT reactions, we combine here large-scale density functional theory (DFT) calculations with classical molecular dynamics (MD) simulations and hybrid quantum mechanics/ molecular mechanics (QM/MM) calculations. We propose a putative mechanism for the PCET reaction and discuss its possible implications in the prevention of ROS formation.

\section{RESULTS AND DISCUSSION}

PCET Energetics between NADH/FMN and the Nearby FeS Centers. In order to study the energetics of the PCET reaction between NADH and FMN, we built DFT and $\mathrm{QM} / \mathrm{MM}$ models of the NADH/FMN site based on the crystal structure of complex I from T. thermophilus, ${ }^{11}$ shown in Figure 2A (see Methods). The stacked aromatic rings of $\mathrm{NADH}$ and FMN in the optimized reactant geometry are at a ca. $3.5 \AA$ distance from each other, with the transferring hydrogen at ca. 2.1 $\AA$ from the acceptor nitrogen. The resulting energy profile for the PCET process is shown in Figure 2B.

We obtain a free energy barrier of ca. $12 \mathrm{kcal} \mathrm{mol}^{-1}$ for the PCET reaction, which is exergonic by ca. $-6 \mathrm{kcal} \mathrm{mol}^{-1}$. The barrier is consistent with the experimental $k_{\text {cat }}$ of ca. 600$15000 \mathrm{~s}^{-1}, 14,15$ which corresponds to a barrier of ca. $12-14$ $\mathrm{kcal} \mathrm{mol}^{-1}$ if a standard pre-exponential factor $k_{\mathrm{B}} T / h \approx 6.45$ $\mathrm{ps}^{-1}$ and a transmission coefficient of $\kappa=1$ are used in transition state theory. We find that the zero-point energy (ZPE) and entropic corrections at $T=310 \mathrm{~K}$ of the model affect the reaction barrier and energetics by $<1 \mathrm{kcal} \mathrm{mol}^{-1}$.

The transferring hydrogen has at the transition state an imaginary frequency of ca. $1380 \mathrm{~cm}^{-1}$, whereas substitution of the hydrogen by deuterium (D) lowers this frequency to ca. $1070 \mathrm{~cm}^{-1}$. This gives a kinetic isotope effect (KIE) of ca. 3.2 at $T=310 \mathrm{~K}$ (see the Supporting Information, SI), whereas quantum corrections increase the KIE to ca. 4.3 (eq 2, SI). These results are consistent with experimental estimates of KIE in complex $\mathrm{I}^{35}$ and are similar to results of other flavoproteins, ${ }^{36}$ suggesting that proton tunneling effects are not significant. ${ }^{37}$

In order to probe how the protein environment influences the PCET energetics, we also studied the reaction by hybrid $\mathrm{QM} / \mathrm{MM}$ calculations. Consistent with the DFT models, we obtain a reaction barrier of ca. $17 \mathrm{kcal} \mathrm{mol}^{-1}$ and a driving force of ca. $-4 \mathrm{kcal} \mathrm{mol}^{-1}$ (Figure 2B). The differences between the DFT and QM/MM models arise from small polarization and structural changes in the surroundings (Figure S15, SI).

Initially, the $\mathrm{NADH}$ and FMN headgroups are neutral, and during the PCET reaction, a net negative charge is transferred to the flavin cofactor (Figure 2B). The transferring proton carries spin but a nearly neutral charge, suggesting that an electron and a proton are transferred concertedly as a hydrogen atom, $\mathrm{H}^{\bullet}$, while the second electron moves along the aromatic ring system of the nucleotides [Figures $2 \mathrm{~A}, \mathrm{~B}$ and S4 (SI)]. We observe a similar $\mathrm{H}^{\bullet}$ transfer process by employing different density functionals, suggesting that the obtained results do not arise from artifacts due to intrinsic DFT errors. This chemical picture is further supported by analysis of frontier orbitals (see Figures S3 and S5-S7 and Movie S1 and Movie S2, SI).

The resulting $\mathrm{FMNH}^{-}$increases the proton affinity of the flavin, which could lead to formation of $\mathrm{FMNH}_{2}$ (cf. refs 38 and 39), for example, via Glu97. However, due to sterical 

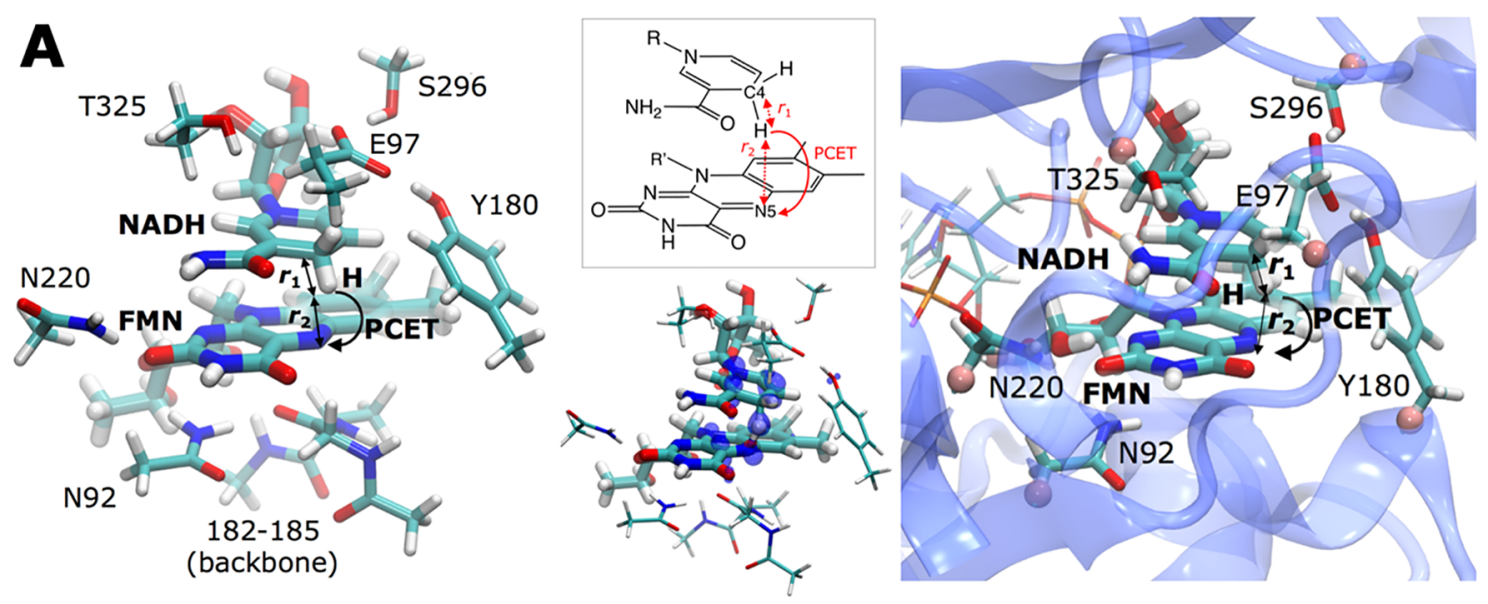

B $\mathrm{NADH}+\mathrm{FMN} \rightarrow \mathrm{NAD}^{+}+\mathrm{FMNH}^{-}$
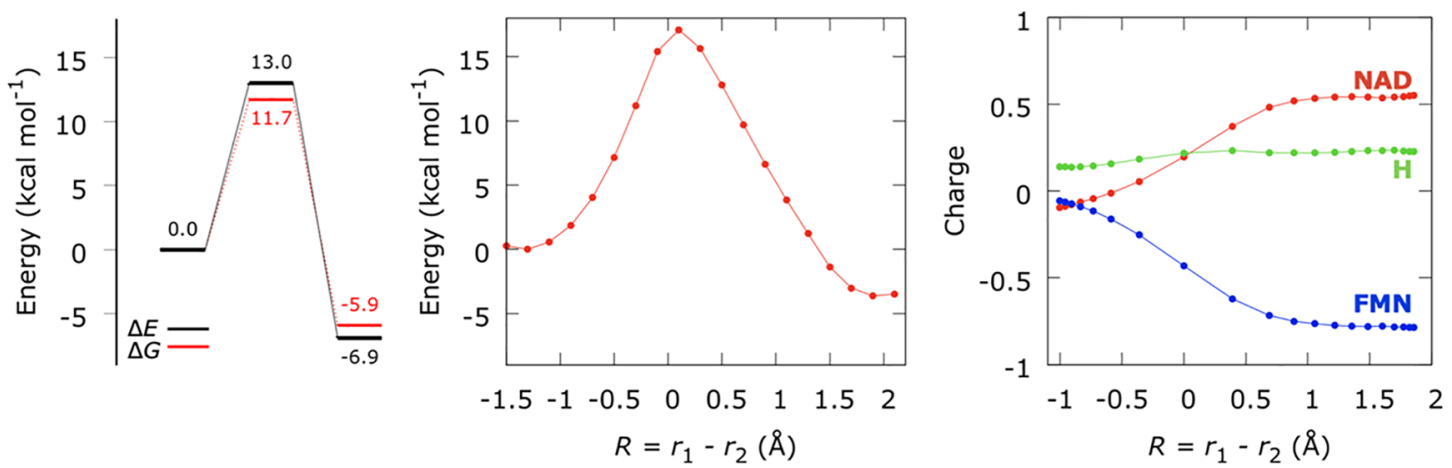

C $[\mathrm{NADH}+\mathrm{FMN}]^{+} \rightarrow \mathrm{NAD}^{+}+\mathrm{FMNH}^{\cdot}$
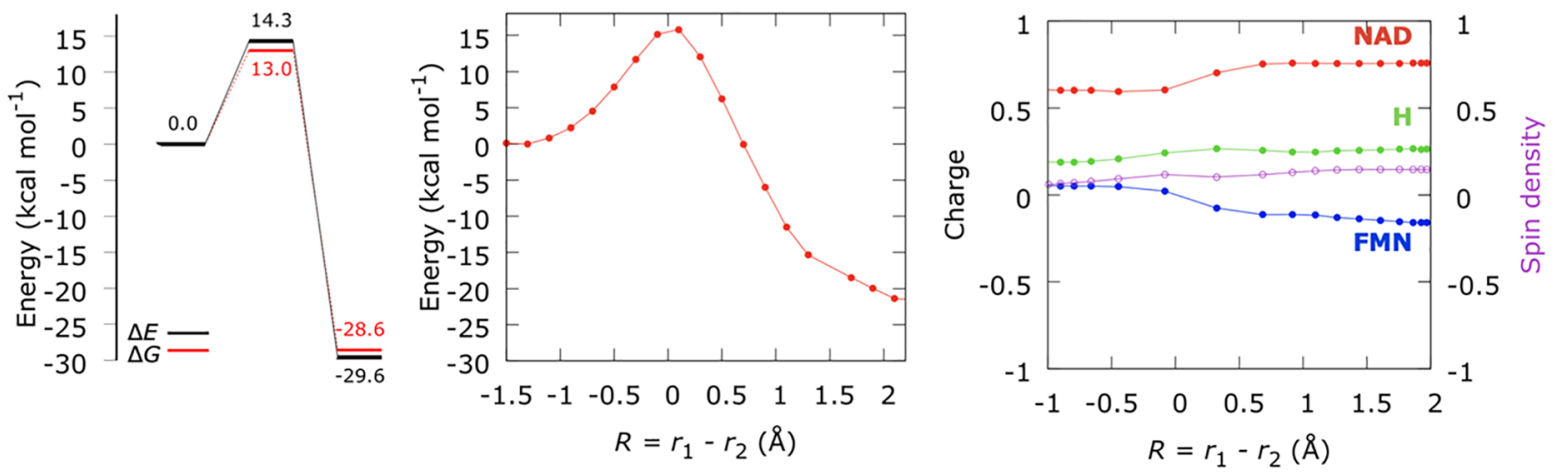

Figure 2. Energetics of the NADH/FMN PCET process. (A) Left: DFT model of the NADH/FMN site. Middle: Schematic representation of $\mathrm{NADH}$ and FMN rings, the proton transfer reaction coordinate, and the spin density distribution of the transition state. Right: QM/MM model of the NADH/FMN site. Link atoms are represented by pink spheres. (B) Energetics of the $\mathrm{H}^{+} / 2 \mathrm{e}^{-}$transfer between NADH and FMN (NADH + $\mathrm{FMN} \rightarrow \mathrm{NAD}^{+}+\mathrm{FMNH}^{-}$). DFT free energy profiles (left), QM/MM energy profile (middle), Mulliken charge distributions (right) on NADH, FMN, and the transferred hydrogen along the reaction path. (C) DFT energetics (left), QM/MM energetics (middle), and charge distributions (right) of the $\mathrm{H}^{+} / \mathrm{e}^{-}$transfer in the oxidized $[\mathrm{NADH} / \mathrm{FMN}]^{+}$system $\left([\mathrm{NADH}+\mathrm{FMN}]^{+} \rightarrow \mathrm{NAD}^{+}+\mathrm{FMNH}^{\bullet}\right)$.

clashes, this process requires $\mathrm{NAD}^{+}$to dissociate away from the binding site, which is expected to take place on the millisecond time scale. ${ }^{15}$ The $\mathrm{FMNH}_{2}$ state was therefore not considered further (but see Supporting Information).

We next probed the proton transfer energetics after oneelectron oxidation of the NADH/FMN pair (Figure 2C), a state that could arise after initial eT to N1a or N3 (see below). This reaction also takes place by $\mathrm{H}^{\bullet}$ transfer but with a slightly higher reaction barrier of ca. $14 \mathrm{kcal} \mathrm{mol}^{-1}$ and an exergonicity of ca. $-30 \mathrm{kcal} \mathrm{mol}^{-1}$. Our $\mathrm{QM} / \mathrm{MM}$ calculations show a similar energy barrier of ca. $16 \mathrm{kcal} \mathrm{mol}^{-1}$, but a somewhat smaller driving force of ca. $-22 \mathrm{kcal} \mathrm{mol}^{-1}$, which is overall similar to the DFT results.

On the basis of these observations, we cannot rule out that the second electron is transferred concertedly to N1a or N3 with the $\mathrm{H}^{\bullet}$ transfer step. We therefore constructed combined models of the NADH/FMN and N1a/N3 subsystems (Figure 3). In the combined DFT models, we also obtain a reaction barrier of ca. $12 \mathrm{kcal} \mathrm{mol}^{-1}$ for the $\mathrm{H}^{\bullet}$ transfer with driving forces of ca. -8 and $-20 \mathrm{kcal} \mathrm{mol}^{-1}$ for the eT to N1a and N3, 


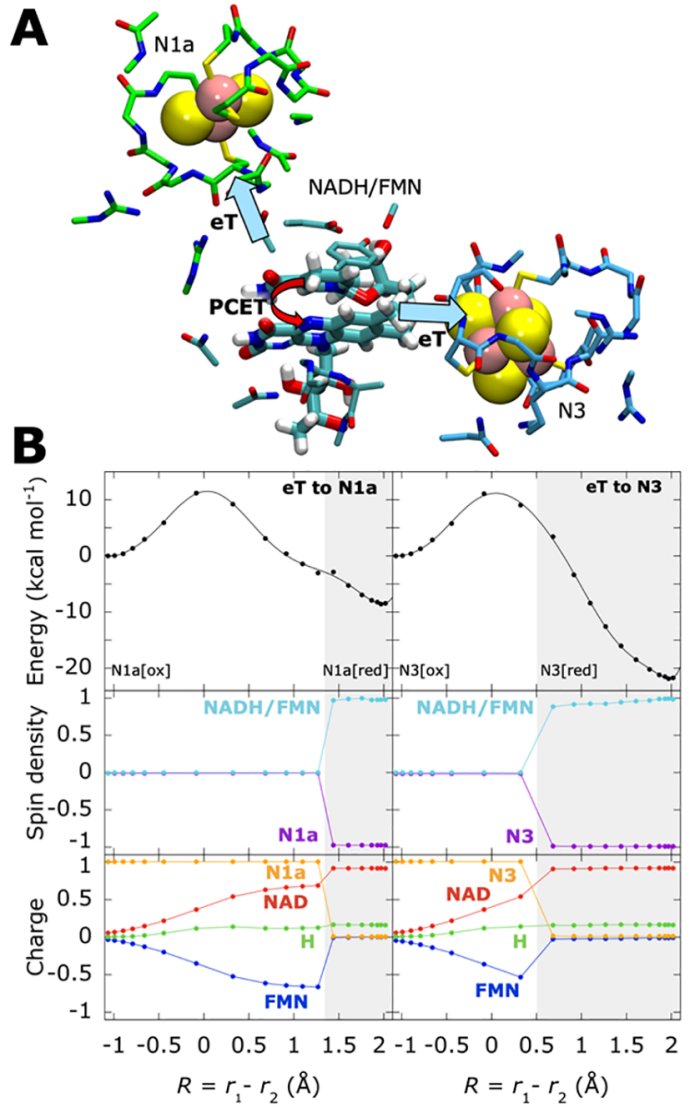

Figure 3. (A) Structure and (B) energetics of the PCET between NADH/FMN and N1a/N3. (B) Top: The PCET between NADH/ FMN and N1a shows that the first electron is transferred concertedly with the hydrogen atom with a barrier of ca. $12 \mathrm{kcal} \mathrm{mol}^{-1}$ and a driving force of ca. $-8 \mathrm{kcal} \mathrm{mol}^{-1}$. The PCET between NADH/FMN and $\mathrm{N} 3$ has a similar barrier but a stronger driving force of ca. -20 $\mathrm{kcal} \mathrm{mol}^{-1}$. Spin (middle panel) and charge (bottom panel) from Mulliken analysis.

respectively. The transferred species also comprises a $\mathrm{H}^{\bullet}$ atom, but the eT to the $\mathrm{FeS}$ center now takes place semiconcertedly with formation of the $\mathrm{FMNH}^{\bullet}$ product state (Figure 3 and Movie S3 and Movie S4, SI).

The DFT calculations suggest that reduction of the N3 center is thermodynamically favored relative to N1a reduction by up to ca. $0.5 \mathrm{eV}$ in the DFT models, but dynamic fluctuations of surrounding charged residues strongly modulate this energetics (see below).

In order to probe approximate nonadiabatic barriers for the eT process, $\Delta G^{\ddagger}=(\Delta G+\lambda)^{2} / 4 \lambda,{ }^{40}$ we computed reorganization energies from the charged localized states. We obtain inner-sphere reorganization energies for the eT from $\mathrm{NAD}^{+}$/ $\mathrm{FMNH}^{-}$to $\mathrm{N} 1 \mathrm{a}$ and $\mathrm{N} 3$ of ca. 0.6 and $0.4 \mathrm{eV}$, respectively, which predicts a nearly barrierless $\Delta G^{\ddagger}$ for the eT to N1a and a somewhat higher $\Delta G^{\ddagger}$ of $0.2 \mathrm{eV}$ for the eT to N3. These findings suggest that the eT to N1a could thus be kinetically slightly favored over the eT to $\mathrm{N} 3$ in bacterial complex I isoforms, despite the latter process being thermodynamically preferred in the structural models.

eT rates also depend on the electronic coupling, which has an exponential dependence on the donor and acceptor distance separation, $\exp \left(-\beta_{0} R_{\mathrm{DA}}\right)$ (see the Supporting Information). We note that the edge-to-edge distances between $\mathrm{NADH}-\mathrm{Nla}$ and $\mathrm{NADH}-\mathrm{N} 3$ are 10.6 and $12.2 \AA$, respectively, whereas the
FMN-N1a and FMN-N3 distances are 11.3 and $6.4 \AA$ (Figure 1), suggesting that the electronic couplings are strong between the $\mathrm{NADH} / \mathrm{N} 1 \mathrm{a}$ and FMN/N3 eT couples. On the basis of the computed eT parameters, a generic proteinpacking density $(\rho=0.76)$, and dynamically averaged edge-toedge distances between the cofactors (see SI and Figure S9), we obtain effective $\mathrm{eT}$ rates in the $1-10 \mathrm{~ns}$ range between the $\mathrm{NADH} / \mathrm{FMN}$ and N1a/N3 couples, further supporting that both $\mathrm{eT}$ processes are fast and possibly competing in the bacterial complex I isoforms considered here.

Conformational Dynamics Coupled to the eT Process. To study how the PCET process is linked to binding of the $\mathrm{NADH} / \mathrm{NAD}^{+}$cofactor, we performed classical $\mathrm{MD}$ simulations of the substrate-bound complex I in different redox states. In simulations of the $\mathrm{NADH} / \mathrm{FMN}$ state with oxidized N1a and N3, the NADH remains tightly stacked with FMN at ca. 3.6 ̊ distance, similar to that in the DFT models and the crystal structure [Figures 2 and S10 (SI)]. After formation of the $\mathrm{NAD}^{+} / \mathrm{FMNH}^{-}$state, the nucleotides also remain in close contact, but at a slightly higher distance of ca. $4.7 \AA$, most likely due to the electrostatically dominant interaction (Figure 4A).

We next probed the dynamics of the $\mathrm{NAD}^{+} / \mathrm{FMNH}^{\bullet}$ state, which is expected to form after eT from $\mathrm{NAD}^{+} / \mathrm{FMNH}^{-}$to one of the nearby FeS centers (see above). With both N1a and $\mathrm{N} 3$ oxidized, a state that could arise if the first electron is transferred via $\mathrm{N} 3$ to $\mathrm{N} 2$, the electrostatic interaction between the cofactors is lost, and $\mathrm{NAD}^{+}$rapidly dissociates from $\mathrm{FMNH}^{\bullet}$. $\mathrm{NAD}^{+}$does not, however, fully leave the binding site but remains bound to conserved aromatic residues on the Nqo1 surface [Figures 4C and S1 (SI)]. We also observe similar dissociation dynamics with the $\mathrm{N} 3$ center modeled in the reduced state (Figure $4 \mathrm{~B}$ ), in which $\mathrm{NAD}^{+}$is fully released to the solvent. However, the lifetime of this state is expected to be transient due to rapid eT from N3 toward the $\mathrm{N} 2$ center, but it may increase upon steady-state turnover conditions when the $\mathrm{FeS}$ is partially reduced. ${ }^{9,29,41} \operatorname{FMN}(\mathrm{H})^{\bullet}$ can also form during reverse eT from $Q$ to FMN. After $\mathrm{NAD}^{+}$ dissociation, our MD simulations suggest that the flavin is accessible to water molecules (Figure S14, SI), and may thus provide a possible pathway for ROS production.

Our calculations suggest that the dynamics of the nearby charged residues may strongly modulate the energetics of the eT to the FeS centers. Arg104 and Arg135 of Nqo1, and Lys121 of Nqo2 move closer to N1a upon its reduction (Figure S8, SI), which in turn increases the redox potential of $\mathrm{N} 1 \mathrm{a}$ and favors the eT from $\mathrm{NAD}^{+} / \mathrm{FMNH}^{-}$. Arg104 and Arg135 of Nqo1 are fully conserved, whereas Lys121 of Nqo2 is not (Figures S1 and S2, SI), which could explain the suggested high variation in the Nla redox potential among different species. $^{24}$ We do not, however, observe that the reduction of the N3 center is coupled to similar conformational changes in surrounding charged residues. These results are thus consistent with a higher inner-sphere reorganization energy for the N1a center relative to N3 (see above).

Interestingly, in stark contrast to the simulations with a reduced $\mathrm{N} 3$, the $\mathrm{NAD}^{+}$remains strongly bound to complex I during the $100 \mathrm{~ns}$ simulation upon reduction of N1a (Figure 4B). In these simulations, Glu97 moves away from N1a and forms an interaction with $\mathrm{NAD}^{+}$(Figure 5B), suggesting that the $\mathrm{N} 1 \mathrm{a}$ reduction may increase the binding affinity of $\mathrm{NAD}^{+}$, consistent with recent observations. ${ }^{31,42}$ These findings also support that the N1a reduction could have a protective role 

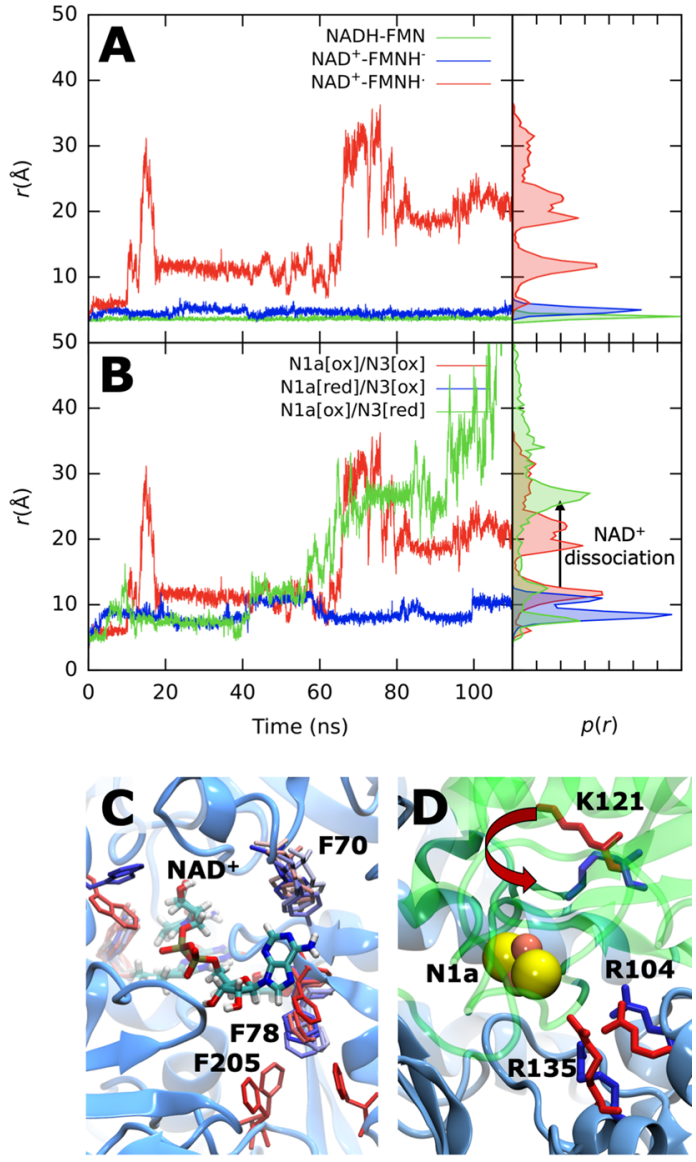

Figure 4. (A) The distance $(r)$ between $\mathrm{NADH}$ and FMN in the $\mathrm{N} 1 \mathrm{a}[\mathrm{ox}] / \mathrm{N} 3[\mathrm{ox}]$ state with $\mathrm{NADH} / \mathrm{FMN}$ (in green), $\mathrm{NAD}^{+} /$ $\mathrm{FMNH}^{-}$(in blue), and $\mathrm{NAD}^{+} / \mathrm{FMNH}^{\bullet}$ (in red). (B) The NADHFMN distance of the $\mathrm{NAD}^{+} / \mathrm{FMNH}^{\bullet}$ with $\mathrm{N} 1 \mathrm{a}[\mathrm{ox}] / \mathrm{N} 3$ [ox] (in red), $\mathrm{N} 1 \mathrm{a}[\mathrm{red}] / \mathrm{N} 3[\mathrm{ox}]$ (in blue), and N1a[ox]/N3[red] (in green). (C) Conserved aromatic residues that form contact with $\mathrm{NAD}^{+}$. (D) Positively charged residues in the proximity of N1a. In panels $\mathrm{C}$ and $\mathrm{D}$, residues shown in red/blue correspond to structures before/after the MD simulations.

against formation of reactive oxygen species that could leak out from FMN. ${ }^{9,29,43}$

Energetics of the Second eT Process. $\mathrm{NAD}^{+}$dissociation decreases the electron affinity of $\mathrm{FMNH}^{\bullet}$ that in turn favors the transfer of the second electron to $\mathrm{N} 3$ by ca. $-3 \mathrm{kcal}$ $\mathrm{mol}^{-1}(130 \mathrm{mV})$ or to N1a by ca. $-1 \mathrm{kcal} \mathrm{mol}^{-1}(50 \mathrm{mV})$ in our DFT models. Experimentally, no flavosemiquinone species has been detected, and we thus expect that the second electron rapidly enters the $\mathrm{FeS}$ chain upon $\mathrm{NAD}^{+}$dissociation. We observe that the oxidation of $\mathrm{FMNH}^{\bullet}$ couples to proton transfer to the conserved Glu97 of Nqo1. After protonation of Glu97, the backward eT from N1a to FMN becomes endergonic by ca. $3 \mathrm{kcal} \mathrm{mol}^{-1}(130 \mathrm{mV})$ in the DFT models, whereas we are not able to oxidize N1a with a singly reduced FMN and a deprotonated Glu97, suggesting that the state has a significantly higher energy. The subsequent reduction of $\mathrm{N} 3$ by FMN with a protonated Glu97 has a strong driving force of ca. $-10 \mathrm{kcal} \mathrm{mol}^{-1}(430 \mathrm{mV})$ in the DFT models. This suggests that deprotonation of FMN by Glu97 could allow for the eT from N1a back to FMN and further to N3, providing a pathway for the second eT toward the N2 center (Figure S12, SI).
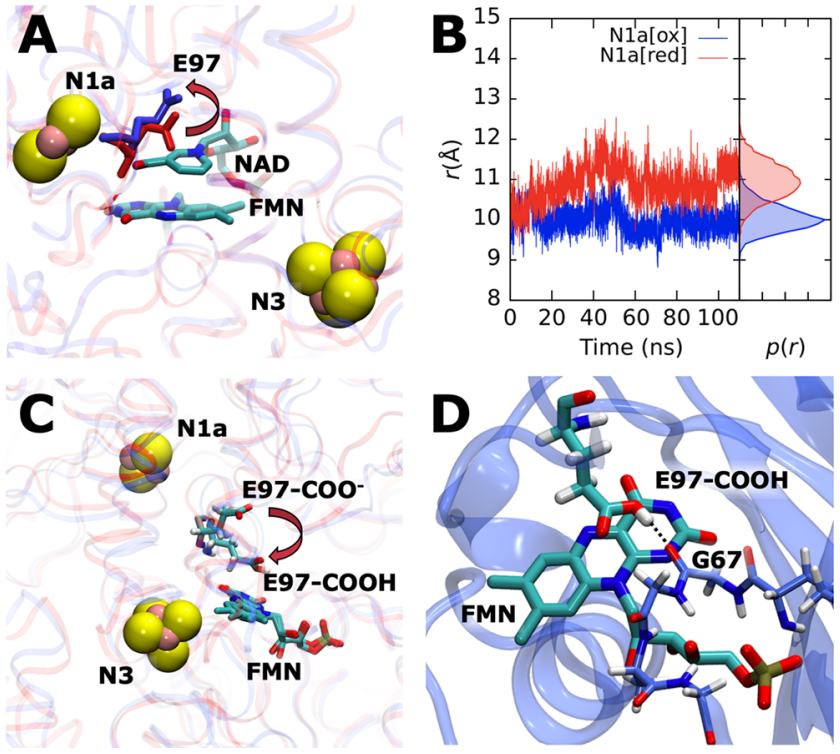

Figure 5. Dynamics of Glu97 in Nqo1. (A) Glu97 moves away from $\mathrm{N} 1 \mathrm{a}$ upon its reduction and closer to $\mathrm{NAD}^{+}$, increasing its binding affinity. (B) The distance $(r)$ between NAD/FMN in different redox states of N1a. (C and D) Dynamics of the protonated Glu97. After the second eT from $\mathrm{FMNH}^{\circ}$, Glu97 protonates, restoring FMN, and forms hydrogen bonds with the backbone of Gly67 in the Nqo1 subunit. This in turn blocks the exposed face of the isoalloxazine ring of FMN and prevents the binding of a new NADH molecule.

When Glu97 is modeled in its protonated state, the barrier for the PCET reaction between NADH/FMN increases by ca. $7 \mathrm{kcal} \mathrm{mol}^{-1}$ (Figure S11, SI), consistent with the experimentally observed decreased $\mathrm{NADH}$-oxidation activity in the E97Q mutant. ${ }^{13}$ Interestingly, the Glu97 protonation alters the conformation of the active site, preventing the entry of a new NADH molecule (Figure $5 \mathrm{C}$ ), by forming a hydrogen bond with the backbone of Gly67 of a Gly-rich loop (residues 66-69) of the Rossmann fold (Figure 5D) that blocks the exposed FMN face where $\mathrm{NADH}$ binds. The proton release from Glu97 to the solvent could therefore favor the initiation of the next catalytic cycle and also provide a mechanism to prevent over-reduction of the FeS chain.

Substitution of Glu97 by a neutral glutamine upshifts the redox potential of the FMN by ca. $40 \mathrm{mV},{ }^{44}$ and mutation of its hydrogen-bonding partner, Tyr180, is linked with development of Leigh syndrome, ${ }^{45}$ a neurometabolic disorder with increased ROS production. In the light of our findings, we suggest that Glu97 has an additional role in modulating $\mathrm{NADH}$ binding $/ \mathrm{NAD}^{+}$release and the transfer of the second electron from N1a further to the FeS chain.

PCET Energetics in Different Complex I Isoforms. To study the PCET reaction energetics and coupled eT in other complex I isoforms, we built DFT models of both E. coli and mouse N1a centers with high- and low-potential forms of the cluster, ${ }^{46,24}$ respectively (see the SI). Our combined models suggest that eT from $\mathrm{NAD}^{+} / \mathrm{FMNH}^{-}$to $E$. coli N1a has similar energetics as in our T. thermophilus models (Figure S13, SI), although the electron affinity is around $80 \mathrm{mV}$ higher in the former model. These findings are consistent with the suggested electron bifurcation to $\mathrm{N} 1 \mathrm{a}$ and $\mathrm{N} 3$ observed in the E. coli complex I. ${ }^{15}$ In contrast, the electron affinity in the mammalian $\mathrm{N} 1$ a center is low, and we do not observe eT to N1a in the models (Figure S13 and Table S3, SI). 


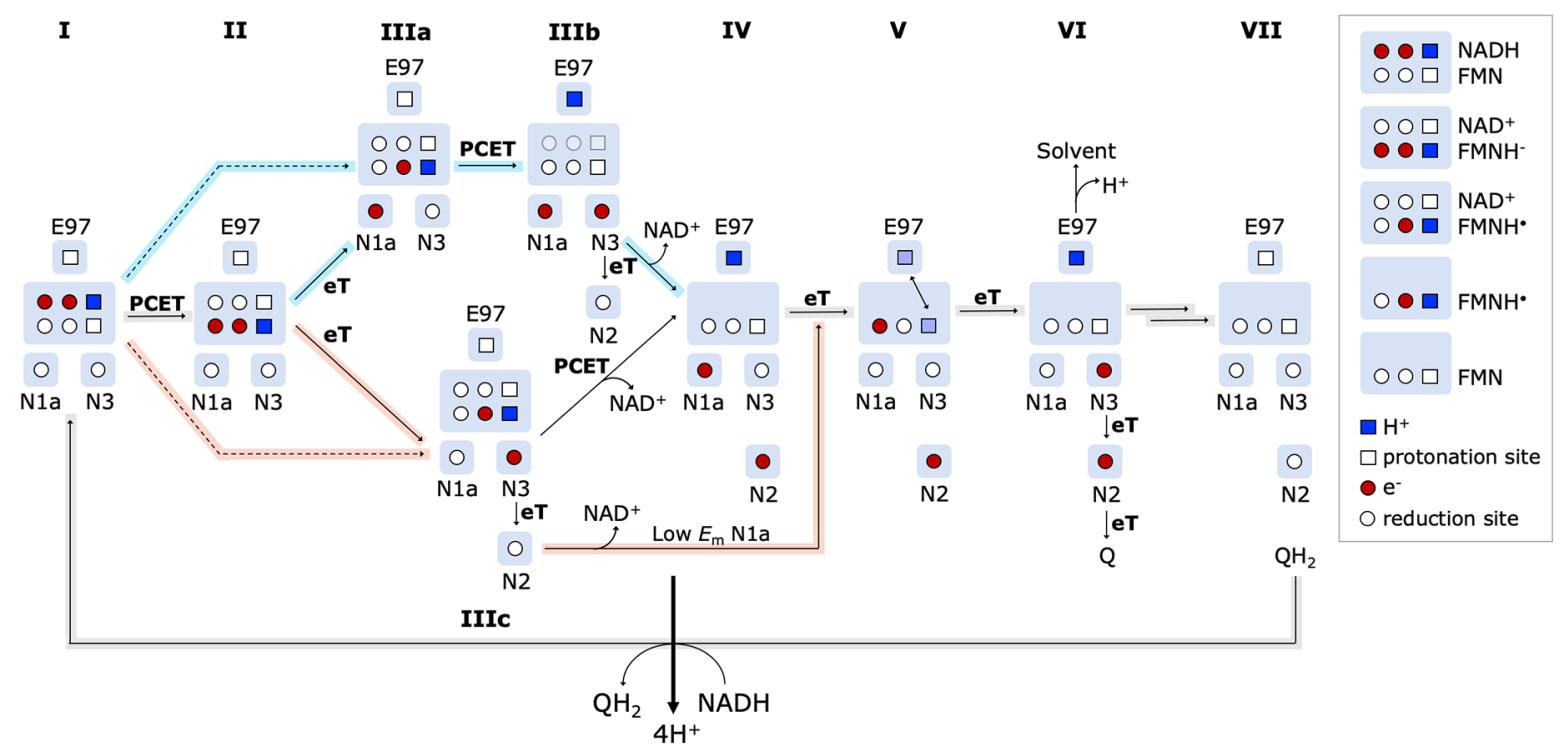

Figure 6. Putative mechanism of the initial PCET steps in complex I. Protonation sites are shown as squares and reduction sites as circles. Highlighted arrows show possible PCET pathways: blue arrows indicate pathways in E. coli-like complex I isoforms, pink arrows show pathways in low-potential N1a isoforms, and gray arrows show pathways that may be common for all complex I isoforms. (I) PCET between NADH and FMN leads to (II) formation of $\mathrm{NAD}^{+} / \mathrm{FMNH}^{-}$that couples to eT to (IIIa) N1a (E. coli-like isoforms), yielding FMNH'. Dashed lines indicate concerted PCET pathways that reduce nearby FeS centers. (IIIb) $\mathrm{NAD}^{+}$partially dissociates from $\mathrm{FMNH}^{\bullet}$ (transparent color) and the second electron is transferred to N3, restoring FMN, which (IV) couples to pT from FMNH ${ }^{\bullet}$ to Glu97. In the competing pathway (IIIc), the first electron is transferred to N3 and onward to N2/Q whereas the second electron is transferred to N1a that couples to NAD ${ }^{+}$dissociation and (IV) pT to Glu97. Complex I isoforms with low-potential $\left(E_{\mathrm{m}}\right)$ N1a are expected to employ the IIIc $\rightarrow$ VI pathway. (V) Protonation of Glu97 increases the electron affinity of FMN, favoring eT from N1a to FMN and (VI) further to N3, which couples to proton release to the solvent. (VII) The second electron at $\mathrm{N} 3$ enters the FeS chain and is transferred to N2, which results in subsequent formation of quinol, $\mathrm{QH}_{2}$. (VII $\left.\rightarrow \mathrm{I}\right)$ The free energy released in quinol formation and dissociation is transduced into pumping protons across the membrane domain.

Interestingly, experiments suggest that the redox potential of Nla could affect whether the reduced flavin leads to production of $\mathrm{H}_{2} \mathrm{O}_{2}$ or superoxide. ${ }^{24,47}$ The bovine complex I with a low-potential N1a mainly produces superoxide, whereas the E. coli complex I with a high potential N1a results in $\mathrm{H}_{2} \mathrm{O}_{2}$ production. ${ }^{47}$ Birrell et al. ${ }^{24}$ did not observe changes in the superoxide $/ \mathrm{H}_{2} \mathrm{O}_{2}$ ratio upon introduction of redoxtuning point mutations near the $\mathrm{Nla}$ cluster in the $24 \mathrm{kDa}$ subunit of the E. coli complex I, whereas Gnandt et al. ${ }^{31}$ observed a decreased ROS production upon N1a reduction. ${ }^{31}$ The molecular basis of these different conditions and isoforms are not fully understood, but taken together, they suggest that N1a reduction could prevent ROS formation, e.g., by increasing $\mathrm{NAD}^{+}$binding affinity, at least in E. coli complex I (see above). The different complex I isoforms have indeed a variation in the $K_{\mathrm{M}}$ of $\mathrm{NADH} / \mathrm{NAD}^{+},{ }^{13,32}$ which in turn could arise from subtle structural differences in the different complex I isoforms and/or from differences in the flavin redox state.

\section{PUTATIVE MECHANISTIC MODEL}

On the basis of our quantum and classical molecular simulations, we propose a mechanistic model for the initial $\mathrm{NADH}$-driven PCET reaction in different complex I isoforms (Figure 6). The PCET reactions are initiated by hydrogen atom transfer that couples to an eT between the NADH/FMN ring systems, forming $\mathrm{NAD}^{+}$and $\mathrm{FMNH}^{-}$. The reaction is exergonic, and it thermodynamically drives the semiconcerted eT from $\mathrm{FMNH}^{-}$to Nla in E. coli-like complex I isoforms, which kinetically competes with reduction of N3 (see below for discussion on low-potential N1a centers). The N1a reduction prevents $\mathrm{NAD}^{+}$from dissociating prematurely from the binding cavity that is favored by formation of $\mathrm{FMNH}^{\bullet}$ due to loss of the electrostatic interaction between $\mathrm{NAD}^{+}$and $\mathrm{FMNH}^{-}$. The electrostatic trapping of $\mathrm{NAD}^{+}$could be important during reverse eT from quinol toward $\mathrm{N} 3$ to prevent electrons from leaking from FMN to the solvent.

The second eT from $\mathrm{FMNH}^{\bullet}$ to $\mathrm{N} 3$ couples to a proton transfer from the flavin to the conserved Glu97. This protonation helps to release the electron stored at N1a to FMN, and further along N3 to the FeS chain. Proton release from Glu97 to the solvent facilitates $\mathrm{NADH}$ binding due to conformational changes, favoring complex I to enter the next catalytic cycle. This protonation-triggered "gate" could prevent over-reduction of the FeS chain, which would also be expected to have a $\mathrm{pH}$-dependence.

The transfer of the two electrons to the nearby FeS centers has been observed in the microseconds time scale in E. coli, whereas $\mathrm{NAD}^{+}$dissociation is reported in the slower milliseconds time scales. ${ }^{15}$ We note that $\mathrm{NAD}^{+}$dissociation favors the release of the second electron that could be triggered by a partial $\mathrm{NAD}^{+}$dissociation from the flavin to the nearby protein surface, as observed in the classical MD simulations (Figure 4).

For complex I isoforms with low-potential N1a centers ${ }^{24,48}$ (see above), the first electron is expected to be transferred to $\mathrm{N} 3$ and further toward the $\mathrm{N} 2$ center. The resulting $\mathrm{FMNH}^{\bullet}$ lowers the affinity for $\mathrm{NAD}^{+}$, which favors dissociation of $\mathrm{NAD}^{+}$to the bulk. Although this also lowers the electron affinity of the flavin and favors release of the second electron, it could lead to formation of a transient dangerous intermediate that may leak electrons out to the solvent. As discussed above, 
ROS is produced differently in mammalian and E. coli complex I. $^{24,47}$

During forward eT to $\mathrm{Q}$ this flavo-semiquinone radical state would be transient due to the fast eT to N3. However, under reverse eT conditions with a highly reduced $\mathrm{FeS}$ chain, the occupation of this state is expected to increase, which in turn would lead to increased ROS generation. ${ }^{9,29,41}$ As discussed above, N1a reduction could prevent ROS formation by trapping $\mathrm{NAD}^{+}$to the binding site under such conditions in E. coli complex I. However, we also note that the reduced N1a decreases the electron affinity of the FMN by $0.3 \mathrm{eV}$ in our DFT models (Table S4, SI) that could further protect against formation of this potentially dangerous state. Complex I variants with a low N1a redox potential may employ, for example, structural changes linked to the active-to-deactive transition ${ }^{49-51}$ to modulate the eT kinetics and prevent ROS formation.

\section{CONCLUDING REMARKS}

In this work, we proposed a mechanistic model for the initial PCET reactions in the NADH/FMN site of respiratory complex I. Our large-scale DFT and QM/MM models that focus here on bacterial complex I isoforms suggest that the PCET between $\mathrm{NADH}$ and FMN involves an exergonic hydrogen atom $\left(\mathrm{H}^{\bullet}\right)$ transfer that couples to eT between the aromatic rings of the cofactors. In our models, the two electrons further bifurcate in the subsequent $\mathrm{eT}$ steps to the nearby $\mathrm{FeS}$ centers, N1a and N3, consistent with EPR experiments $^{15}$ and with mechanistic analogy to other flavoenzymes. ${ }^{52}$ The N1a reduction increases the binding affinity of $\mathrm{NAD}^{+}$that prevents the electrons from leaking out to the solvent. Proton transfer between the transiently formed $\mathrm{FMNH}^{\bullet}$ species and a conserved Glu97 was found to facilitate the release of the second electron from the flavin and to prevent a new $\mathrm{NADH}$ from entering the binding site by triggering conformational changes in the cavity. We suggest that this proton transfer process is also involved in releasing the electron stored on the N1a center via FMN back to the FeS chain. These findings also imply that the forward and backward $\mathrm{eT}$ reactions could be modulated by the external $\mathrm{pH}$.

Our calculations showed that the protein dynamics may strongly influence the energetics of the eT between $\mathrm{NADH}$ / FMN and the N1a/N3 centers. Conformational changes in highly conserved charged residues of Nqo1 that move closer to the $\mathrm{FeS}$ centers facilitate their reduction, whereas the high variability of charged residues in the $\mathrm{Nqo} 2$ subunit, as also observed from large variations in the $\mathrm{Nla}$ redox potential in different species, i.e., from $-240 \mathrm{mV}$ in $E$. coli to suggested values of ca. $-400 \mathrm{mV}$ in mammalian complex $\mathrm{I}^{24}$ is consistent with our calculations on different complex I isoforms. In DFT models of low-potential N1a isoforms, we do not observe reduction of $\mathrm{N} 1 \mathrm{a}$, suggesting that $\mathrm{eT}$ follows a different reaction pathway, as highlighted in Figure 6. Modulation of the eT kinetics might couple to conformational changes, which are currently not well understood. Redox-triggered conformational changes have not yet been observed in available experimental structures, possibly due to their $3-4 \AA$ resolution. Taken together, our combined results suggest that the long-range electron transfer process in complex I is initiated by coupled protonation and reduction steps in the NADH/FMN site that trigger electrostatic and conformational changes in the protein environment to prevent electron leaks and over-reduction of the FeS chain. The model has important mechanistic implications for the forward and reverse electron transfer process in complex I and for understanding the subsequent quinone reduction process that, in turn, activates the proton pumping machinery.

\section{METHODOLOGY}

DFT models for the NADH/FMN, N1a, and N3 subsystems comprising first- and second sphere protein residues were built on the basis of the crystal structure of complex I from T. thermophilus (PDB ID: 3IAM; see the Supporting Information). ${ }^{11}$ Additional N1a models from E. coli and mammalian complex I were also built (see the SI). The individual subsystems comprised 150-170 atoms and the combined systems ca. 330-350 atoms. Spin energetics were treated using the spin-flip broken symmetry approach (Table S2, SI), ${ }^{53,54}$ and geometry optimizations were performed at the B3LYP-D3/def2-SVP/ $\operatorname{def2}-\mathrm{TZVP}(\mathrm{Fe}) / \varepsilon=4$ level, with single-point energies reported at the B3LYP-D3/def2-TZVP $/ \varepsilon=4$ level. ${ }^{59-59}$ The electronic structures of the combined models were converged into localized "diabatic" electronic states by merging the molecular orbitals of the electron donor $(\mathrm{NADH} / \mathrm{FMN})$ or acceptor sites (N1a or N3) in their respective reduced/oxidized states together. Zero-point energies (ZPE) and entropic corrections were estimated at the B3LYP-D3/ def2-SVP $/ \varepsilon=4$ level using smaller model systems. Reaction pathways for the PCET reaction were optimized using a chain-of-state method, ${ }^{60}$ and hydrogen/deuterium (H/D) kinetic isotope effects (KIE) were estimated on the basis of the ZPE differences between reactant and transition state structures. Approximate quantum effects were evaluated by the Wigner correction, ${ }^{61}$ and electron transfer rates were based on the Moser-Dutton ruler. ${ }^{62}$ All QM calculations were performed with TURBOMOLE versions 6.6-7.2. ${ }^{63}$

$\mathrm{QM} / \mathrm{MM}$ models of the NADH/FMN subsystem with $132 \mathrm{QM}$ atoms were constructed for computing the potential energy surface (PES) for the PCET reaction between NADH and FMN. The QM subsystem was treated at the B3LYP-D3/def2-SVP level, and the MM subsystem was described at the CHARMM36 level based on the classical model (see below). ${ }^{64}$ The reaction coordinate, $R=r_{1}-r_{2}$ where $r_{1}=(\mathrm{NADH}: \mathrm{C} 4-\mathrm{H})$ and $r_{2}=(\mathrm{FMN}: \mathrm{N} 5-\mathrm{H})$, was optimized from reactants, $R=-1.5 \AA$, to products, $R=+2.5 \AA$, using a harmonic potential restrain. During optimization, the $\mathrm{QM}$ region and the $\mathrm{MM}$ surroundings within a $15 \AA$ sphere centered on FMN were allowed to relax. All QM/MM calculations were performed with the CHARMM/ TURBOMOLE interface. ${ }^{65}$

Classical atomistic molecular dynamics (MD) simulations were performed on the hydrophilic domain of complex I by including subunits Nqo1, with the NADH/FMN cofactors, Nqo2, Nqo3, and Nqo15. The protein was solvated and neutralized in a TIP3P water/ $100 \mathrm{mM} \mathrm{NaCl}$ solvation box and comprised ca. 170000 atoms. MD simulations were performed at $p=1 \mathrm{bar}$ and $T=310 \mathrm{~K}$ in an NPT ensemble, using a $2 \mathrm{fs}$ integration time step and treating long-range electrostatic interactions by the Particle Mesh Ewald (PME) method. The CHARMM36 force field ${ }^{66}$ and our in-house DFT-derived parameters for N1a and $\mathrm{N} 3$ in both oxidized and reduced states were used. The MD simulations were performed with NAMD2, ${ }^{67}$ and VMD was used for analysis. ${ }^{68}$ All simulations are summarized in Table S1 (SI). See the Supporting Information for a detailed computational description.

\section{ASSOCIATED CONTENT}

\section{Supporting Information}

The Supporting Information is available free of charge on the ACS Publications website at DOI: 10.1021/jacs.8b11059.

Detailed computational methods; figures showing the multiple sequence alignment of Nqo1/NuoF/NDUFV1 and Nqo2/NuoE/NDUFV2 subunits of complex I, energetics of the NADH/FMN PCET process, radial distribution of the spin density on the transferred hydrogen atom, spin density on the transition state 
structures, spin density and HOMO orbitals of the reactant, the transition state, and the product structure, distances for $\mathrm{N} 1 \mathrm{a}$ in $\mathrm{Nqo} 1$ and $\mathrm{Nqo} 2$ subunits, edge-toedge distances between NADH-N1a/N3 and FMNN1a/N3 during MD simulations, dissociation energy profiles, energetics of the PCET reactions, the energetics of the second electron transfer from the flavin to the FeS centers from DFT models, energetics of the PCET reaction between $\mathrm{NADH} / \mathrm{FMN}$ and N1a in complex I, the FMN binding cavity, comparison of the energetics of the NADH/FMN PCET reaction between QM and $\mathrm{QM} / \mathrm{MM}$ levels; tables summarizing the simulations, optimized broken-symmetry spin-flip states for the oxidized and reduced $\mathrm{FeS}$ centers, electron affinities of the N1a FeS in DFT models, electron affinities of FMN with oxidized and reduced N1a; and captions describing Movies S1-S4 (PDF)

Movie S1: Spin density in the PCET reaction between $\mathrm{NADH}$ and FMN (MPG)

Movie S2: Highest occupied molecular orbital (HOMO) in the PCET reaction between $\mathrm{NADH}$ and FMN (MPG)

Movie S3: Spin density in the PCET reaction between $\mathrm{NADH}$ and FMN that couples eT to N1a (MPG)

Movie S4: Spin density in the PCET reaction between $\mathrm{NADH}$ and FMN that couples eT to N3 (MPG)

\section{AUTHOR INFORMATION}

\section{Corresponding Author}

*ville.kaila@ch.tum.de

\section{ORCID}

Ville R. I. Kaila: 0000-0003-4464-6324

\section{Notes}

The authors declare no competing financial interest.

\section{ACKNOWLEDGMENTS}

This work received funding from the European Research Council (ERC) under the European Union's Horizon 2020 research and innovation program/grant agreement 715311 . The Leibniz-Rechenzentrum (LRZ) (project pr48de) provided computational resources.

\section{REFERENCES}

(1) Brandt, U. Energy Converting NADH: Quinone Oxidoreductase (Complex I). Annu. Rev. Biochem. 2006, 75 (1), 69-92.

(2) Wikström, M. Two Protons Are Pumped from the Mitochondrial Matrix per Electron Transferred between $\mathrm{NADH}$ and Ubiquinone. FEBS Lett. 1984, 169 (2), 300-304.

(3) Verkhovskaya, M.; Bloch, D. A. Energy-Converting Respiratory Complex I: On the Way to the Molecular Mechanism of the Proton Pump. Int. J. Biochem. Cell Biol. 2013, 45 (2), 491-511.

(4) Hirst, J. Mitochondrial Complex I. Annu. Rev. Biochem. 2013, 82 (1), 551-575.

(5) Kaila, V. R. I. Long-Range Proton-Coupled Electron Transfer in Biological Energy Conversion: Towards Mechanistic Understanding of Respiratory Complex I. J. R. Soc., Interface 2018, 15 (141), 20170916.

(6) Mitchell, P. Coupling of Phosphorylation to Electron and Hydrogen Transfer by a Chemi-Osmotic Type of Mechanism. Nature 1961, 191 (4784), 144-148.

(7) Yoshida, M.; Muneyuki, E.; Hisabori, T. ATP Synthase - A Marvellous Rotary Engine of the Cell. Nat. Rev. Mol. Cell Biol. 2001, 2 (9), 669-677.
(8) Lambert, A. J.; Brand, M. D. Superoxide Production by NADH:Ubiquinone Oxidoreductase (Complex I) Depends on the PH Gradient across the Mitochondrial Inner Membrane. Biochem. J. 2004, 382 (2), 511-517.

(9) Pryde, K. R.; Hirst, J. Superoxide Is Produced by the Reduced Flavin in Mitochondrial Complex I: A Single, Unified Mechanism That Applies during Both Forward and Reverse Electron Transfer. J. Biol. Chem. 2011, 286 (20), 18056-18065.

(10) Murphy, M. P. How Mitochondria Produce Reactive Oxygen Species. Biochem. J. 2009, 417 (1), 1-13.

(11) Berrisford, J. M.; Sazanov, L. A. Structural Basis for the Mechanism of Respiratory Complex I. J. Biol. Chem. 2009, 284 (43), 29773-29783.

(12) Baradaran, R.; Berrisford, J. M.; Minhas, G. S.; Sazanov, L. A. Crystal Structure of the Entire Respiratory Complex I. Nature 2013, 494 (7438), 443-448.

(13) Euro, L.; Belevich, G.; Bloch, D. A.; Verkhovsky, M. I.; Wikström, M.; Verkhovskaya, M. The Role of the Invariant Glutamate 95 in the Catalytic Site of Complex I from Escherichia Coli. Biochim. Biophys. Acta, Bioenerg. 2009, 1787 (1), 68-73.

(14) Birrell, J. A.; Yakovlev, G.; Hirst, J. Reactions of the Flavin Mononucleotide in Complex I: A Combined Mechanism Describes NADH Oxidation Coupled to the Reduction of APAD+, Ferricyanide, or Molecular Oxygen. Biochemistry 2009, 48 (50), 12005-12013.

(15) Verkhovskaya, M. L.; Belevich, N. P.; Euro, L.; Wikström, M.; Verkhovsky, M. I. Real-Time Electron Transfer in Respiratory Complex I. Proc. Natl. Acad. Sci. U. S. A. 2008, 105 (10), 3763-3767.

(16) Hirst, J. Towards the Molecular Mechanism of Respiratory Complex I. Biochem. J. 2010, 425 (2), 327-339.

(17) Mayer, J. M.; Hrovat, D. A.; Thomas, J. L.; Borden, W. T. Proton-Coupled Electron Transfer versus Hydrogen Atom Transfer in Benzyl/Toluene, Methoxyl/Methanol, and Phenoxyl/Phenol SelfExchange Reactions. J. Am. Chem. Soc. 2002, 124 (37), 11142-11147.

(18) Morris, W. D.; Mayer, J. M. Separating Proton and Electron Transfer Effects in Three-Component Concerted Proton-Coupled Electron Transfer Reactions. J. Am. Chem. Soc. 2017, 139 (30), 10312-10319.

(19) Kaila, V. R. I.; Hummer, G. Energetics of Direct and WaterMediated Proton-Coupled Electron Transfer. J. Am. Chem. Soc. 2011, 133 (47), 19040-19043.

(20) Hammes-Schiffer, S.; Stuchebrukhov, A. A. Theory of Coupled Electron and Proton Transfer Reactions. Chem. Rev. 2010, 110 (12), 6939-6960.

(21) Gamiz-Hernandez, A. P.; Jussupow, A.; Johansson, M. P.; Kaila, V. R. I. Terminal Electron-Proton Transfer Dynamics in the Quinone Reduction of Respiratory Complex I. J. Am. Chem. Soc. 2017, 139 (45), 16282-16288.

(22) Sharma, V.; Belevich, G.; Gamiz-Hernandez, A. P.; Róg, T.; Vattulainen, I.; Verkhovskaya, M. L.; Wikström, M.; Hummer, G.; Kaila, V. R. I. Redox-Induced Activation of the Proton Pump in the Respiratory Complex I. Proc. Natl. Acad. Sci. U. S. A. 2015, 112 (37), 11571-11576.

(23) Ohnishi, T. Iron-Sulfur Clusters/Semiquinones in Complex I. Biochim. Biophys. Acta, Bioenerg. 1998, 1364 (2), 186-206.

(24) Birrell, J. A.; Morina, K.; Bridges, H. R.; Friedrich, T.; Hirst, J. Investigating the Function of $[2 \mathrm{Fe}-2 \mathrm{~S}]$ Cluster $\mathrm{N} 1 \mathrm{a}$, the off-Pathway Cluster in Complex I, by Manipulating Its Reduction Potential. Biochem. J. 2013, 456 (1), 139-146.

(25) Barker, C. D.; Reda, T.; Hirst, J. The Flavoprotein Subcomplex of Complex I (NADH:Ubiquinone Oxidoreductase) from Bovine Heart Mitochondria: Insights into the Mechanisms of $\mathrm{NADH}$ Oxidation and NAD+ Reduction from Protein Film Voltammetry. Biochemistry 2007, 46 (11), 3454-3464.

(26) Zwicker, K.; Galkin, A.; Dröse, S.; Grgic, L.; Kerscher, S.; Brandt, U. The Redox-Bohr Group Associated with Iron-Sulfur Cluster N2 of Complex I. J. Biol. Chem. 2006, 281 (32), 2301323017. 
(27) Hirst, J.; Roessler, M. M. Energy Conversion, Redox Catalysis and Generation of Reactive Oxygen Species by Respiratory Complex I. Biochim. Biophys. Acta, Bioenerg. 2016, 1857 (7), 872-883.

(28) De Vries, S.; Dörner, K.; Strampraad, M. J. F.; Friedrich, T. Electron Tunneling Rates in Respiratory Complex I Are Tuned for Efficient Energy Conversion. Angew. Chem., Int. Ed. 2015, 54 (9), 2844-2848.

(29) Kussmaul, L.; Hirst, J. The Mechanism of Superoxide Production by NADH:Ubiquinone Oxidoreductase (Complex I) from Bovine Heart Mitochondria. Proc. Natl. Acad. Sci. U. S. A. 2006, 103 (20), 7607-7612.

(30) Cochemé, H. M.; Murphy, M. P. Complex I Is the Major Site of Mitochondrial Superoxide Production by Paraquat. J. Biol. Chem. 2008, 283 (4), 1786-1798.

(31) Gnandt, E.; Schimpf, J.; Harter, C.; Hoeser, J.; Friedrich, T. Reduction of the Off-Pathway Iron-Sulphur Cluster N1a of Escherichia Coli Respiratory Complex I Restrains $\mathrm{NAD}^{+}$Dissociation. Sci. Rep. 2017, 7 (1), 8754.

(32) Grivennikova, V. G.; Roth, R.; Zakharova, N. V.; Hägerhäll, C.; Vinogradov, A. D. The Mitochondrial and Prokaryotic ProtonTranslocating NADH:Ubiquinone Oxidoreductases: Similarities and Dissimilarities of the Quinone-Junction Sites. Biochim. Biophys. Acta, Bioenerg. 2003, 1607 (2-3), 79-90.

(33) Kotlyar, A. B.; Borovok, N. NADH Oxidation and $\mathrm{NAD}^{+}$ Reduction Catalysed by Tightly Coupled inside-out Vesicles from Paracoccus Denitrificans. Eur. J. Biochem. 2002, 269 (16), 40204024.

(34) Sled, V. D.; Vinogradov, A. D. Kinetics of the Mitochondrial NADH-Ubiquinone Oxidoreductase Interaction with Hexammineruthenium(III). Biochim. Biophys. Acta, Bioenerg. 1993, 1141 (2-3), 262-268.

(35) Birrell, J. A.; Hirst, J. Investigation of NADH Binding, Hydride Transfer, and $\mathrm{NAD}+$ Dissociation during $\mathrm{NADH}$ Oxidation by Mitochondrial Complex i Using Modified Nicotinamide Nucleotides. Biochemistry 2013, 52 (23), 4048-4055.

(36) Pang, J.; Hay, S.; Scrutton, N. S.; Sutcliffe, M. J. Deep Tunneling Dominates the Biologically Important Hydride Transfer Reaction from NADH to FMN in Morphinone Reductase. J. Am. Chem. Soc. 2008, 130 (22), 7092-7097.

(37) Kiefer, P. M.; Hynes, J. T. Theoretical Aspects of Tunneling Proton Transfer Reactions in a Polar Environment. J. Phys. Org. Chem. 2010, 23 (7), 632-646.

(38) Sled, V. D.; Rudnitzky, N. I.; Ohnishi, T.; Hatefi, Y. Thermodynamic Analysis of Flavin in Mitochondrial NADH:Ubiquinone Oxidoreductase (Complex I). Biochemistry 1994, 33 (33), 10069-10075

(39) Holt, P. J.; Efremov, R. G.; Nakamaru-Ogiso, E.; Sazanov, L. A. Reversible FMN Dissociation from Escherichia Coli Respiratory Complex I. Biochim. Biophys. Acta, Bioenerg. 2016, 1857 (11), 17771785.

(40) Marcus, R. A.; Sutin, N. Electron Transfers in Chemistry and Biology. Biochim. Biophys. Acta, Rev. Bioenerg. 1985, 811 (3), 265322.

(41) Robb, E. L.; Hall, A. R.; Prime, T. A.; Eaton, S.; Szibor, M.; Viscomi, C.; James, A. M.; Murphy, M. P. Control of Mitochondrial Superoxide Production by Reverse Electron Transport at Complex I. J. Biol. Chem. 2018, 293 (25), 9869-9879.

(42) Na, S.; Jurkovic, S.; Friedrich, T.; Koslowski, T. Charge Transfer through a Fragment of the Respiratory Complex I and Its Regulation: An Atomistic Simulation Approach. Phys. Chem. Chem. Phys. 2018, 20 (30), 20023-20032.

(43) Hirst, J.; King, M. S.; Pryde, K. R. The Production of Reactive Oxygen Species by Complex I. Biochem. Soc. Trans. 2008, 36 (5), 976-980.

(44) Euro, L.; Belevich, G.; Wikström, M.; Verkhovskaya, M. High Affinity Cation-Binding Sites in Complex I from Escherichia Coli. Biochim. Biophys. Acta, Bioenerg. 2009, 1787 (8), 1024-1028.

(45) Varghese, F.; Atcheson, E.; Bridges, H. R.; Hirst, J. Characterization of Clinically Identified Mutations in NDUFV1, the
Flavin-Binding Subunit of Respiratory Complex I, Using a Yeast Model System. Hum. Mol. Genet. 2015, 24 (22), 6350-6360.

(46) Agip, A. N. A.; Blaza, J. N.; Bridges, H. R.; Viscomi, C.; Rawson, S.; Muench, S. P.; Hirst, J. Cryo-EM Structures of Complex I from Mouse Heart Mitochondria in Two Biochemically Defined States. Nat. Struct. Mol. Biol. 2018, 25 (7), 548-556.

(47) Esterházy, D.; King, M. S.; Yakovlev, G.; Hirst, J. Production of Reactive Oxygen Species by Complex I (NADH:Ubiquinone Oxidoreductase) from Escherichia Coli and Comparison to the Enzyme from Mitochondria. Biochemistry 2008, 47 (12), 3964-3971.

(48) Zu, Y.; Di Bernardo, S.; Yagi, T.; Hirst, J. Redox Properties of the $[2 \mathrm{Fe}-2 \mathrm{~S}]$ Center in the $24 \mathrm{KDa}$ (NQO2) Subunit of NADH:Ubiquinone Oxidoreductase (Complex I). Biochemistry 2002, 41 (31), 10056-10069.

(49) Zhu, J.; Vinothkumar, K. R.; Hirst, J. Structure of Mammalian Respiratory Complex I. Nature 2016, 536 (7616), 354-358.

(50) Fiedorczuk, K.; Letts, J. A.; Degliesposti, G.; Kaszuba, K.; Skehel, M.; Sazanov, L. A. Atomic Structure of the Entire Mammalian Mitochondrial Complex I. Nature 2016, 538 (7625), 406-410.

(51) Di Luca, A.; Kaila, V. R. I. Global Collective Motions in the Mammalian and Bacterial Respiratory Complex I. Biochim. Biophys. Acta, Bioenerg. 2018, 1859 (5), 326-332.

(52) Buckel, W.; Thauer, R. K. Flavin-Based Electron Bifurcation, A New Mechanism of Biological Energy Coupling. Chem. Rev. 2018, 118 (7), 3862-3886.

(53) Mouesca, J. M.; Noodleman, L.; Case, D. A. Density-Functional Calculations of Spin Coupling in $[\mathrm{Fe} 4 \mathrm{~s} 4](3+)$ Clusters. Int. J. Quantum Chem. 1995, 56 (S22), 95-102.

(54) Noodleman, L.; Case, D. A.; Aizman, A. Broken Symmetry Analysis of Spin Coupling in Iron-Sulfur Clusters. J. Am. Chem. Soc. 1988, 110 (4), 1001-1005.

(55) Becke, A. D. Density-Functional Thermochemistry. III. The Role of Exact Exchange. J. Chem. Phys. 1993, 98 (7), 5648-5652.

(56) Lee, C.; Yang, W.; Parr, R. G. Development of the ColleSalvetti Correlation-Energy Formula into a Functional of the Electron Density. Phys. Rev. B: Condens. Matter Mater. Phys. 1988, 37 (2), 785-789.

(57) Schäfer, A.; Horn, H.; Ahlrichs, R. Fully Optimized Contracted Gaussian Basis Sets for Atoms Li to Kr. J. Chem. Phys. 1992, 97 (4), $2571-2577$

(58) Grimme, S.; Antony, J.; Ehrlich, S.; Krieg, H. A Consistent and Accurate $\mathrm{Ab}$ Initio Parametrization of Density Functional Dispersion Correction (DFT-D) for the 94 Elements H-Pu. J. Chem. Phys. 2010, 132 (15), 154104-1-19.

(59) Klamt, A.; Schüurmann, G. COSMO: A New Approach to Dielectric Screening in Solvents with Explicit Expressions for the Screening Energy and Its Gradient. J. Chem. Soc., Perkin Trans. 2 1993, No. 5, 799-805.

(60) Plessow, P. Reaction Path Optimization without NEB Springs or Interpolation Algorithms. J. Chem. Theory Comput. 2013, 9 (3), $1305-1310$.

(61) Bell, R. P. Liversidge Lecture: Recent Advances in the Study of Kinetic Hydrogen Isotope Effects. Chem. Soc. Rev. 1974, 3 (4), 513544

(62) Page, C. C.; Moser, C. C.; Chen, X.; Dutton, P. L. Natural Engineering Principles of Electron Tunnelling in Biological Oxidation-Reduction. Nature 1999, 402 (6757), 47-52.

(63) Ahlrichs, R.; Bär, M.; Häser, M.; Horn, H.; Kölmel, C. Electronic Structure Calculations on Workstation Computers: The Program System Turbomole. Chem. Phys. Lett. 1989, 162 (3), 165169.

(64) Huang, J.; Rauscher, S.; Nawrocki, G.; Ran, T.; Feig, M.; De Groot, B. L.; Grubmüller, H.; MacKerell, A. D. CHARMM36m: An Improved Force Field for Folded and Intrinsically Disordered Proteins. Nat. Methods 2017, 14 (1), 71-73.

(65) Riahi, S.; Rowley, C. N. The CHARMM-TURBOMOLE Interface for Efficient and Accurate QM/MM Molecular Dynamics, Free Energies, and Excited State Properties. J. Comput. Chem. 2014, 35 (28), 2076-2086. 
(66) Huang, J.; Mackerell, A. D. CHARMM36 All-Atom Additive Protein Force Field: Validation Based on Comparison to NMR Data. J. Comput. Chem. 2013, 34 (25), 2135-2145.

(67) Phillips, J. C.; Braun, R.; Wang, W.; Gumbart, J.; Tajkhorshid, E.; Villa, E.; Chipot, C.; Skeel, R. D.; Kalé, L.; Schulten, K. Scalable Molecular Dynamics with NAMD. J. Comput. Chem. 2005, 26 (16), 1781-1802.

(68) Humphrey, W.; Dalke, A.; Schulten, K. VMD: Visual Molecular Dynamics. J. Mol. Graphics 1996, 14 (1), 33-38. 